\title{
Cadmium Disrupts Vestibular Function by Interfering with Otolith Formation.
}

\author{
Adrian J. Green ${ }^{1}$, Carolyn J. Mattingly ${ }^{1,2}$, and Antonio Planchart ${ }^{1,2}$ \\ ${ }^{1}$ Department of Biological Sciences and the \\ ${ }^{2}$ Center for Human Health and the Environment, North Carolina State University, \\ Raleigh, NC
}




\section{Abstract}

3 Cadmium $\left(\mathrm{Cd}^{2+}\right)$ is a transition metal found ubiquitously in the earth's crust and is

4 extracted in the production of other metals such as copper, lead, and zinc ${ }^{1,2}$. Human

5 exposure to $\mathrm{Cd}^{2+}$ occurs through food consumption, cigarette smoking, and the

6 combustion of fossil fuels. $\mathrm{Cd}^{2+}$ has been shown to be nephrotoxic, neurotoxic, and

7 osteotoxic, and is a known carcinogen. Animal studies and epidemiological studies have

8 linked prenatal $\mathrm{Cd}^{2+}$ exposure to hyperactivity and balance disorders although the

9 mechanisms remain unknown. In this study we show that zebrafish developmentally exposed to $\mathrm{Cd}^{2+}$ exhibit abnormal otolith development and show an increased tendency

11 to swim in circles, observations that are consistent with an otolith-mediated vestibular

12 defect, in addition to being hyperactive. We also demonstrate that the addition of

13 calcium rescues otolith malformation and reduces circling behavior but has no

14 ameliorating effect on hyperactivity, suggesting that hyperactivity and balance disorders

15 in human populations exposed to $\mathrm{Cd}$ are manifestations of separate underlying

16 molecular pathways. 


\section{Introduction}

The vestibular system of the vertebrate inner ear consists of three semicircular canals harboring sensory epithelia that respond to rotational acceleration. Two sensory organs, the utricle and saccule, which sense linear acceleration and gravity ${ }^{3,4}$ are located at the base of the semicircular canals. Together with the central nervous system, the vestibular system integrates both the magnitude and direction of head movements about an axis. The utricle and saccule house otoconia (termed otoliths in fish), which are bio-crystals composed of calcium carbonate $\left(\mathrm{CaCO}_{3}\right)$ and proteins ${ }^{3,4}$, that lie above the ciliated hair cells of the sensory epithelium (macula); they are anchored to the hair cells by a honeycomb-like otoconial membrane ${ }^{3}$. If this membrane is compromised, a common occurrence in individuals with Ménière's disease ${ }^{5}$, the otoconia can detach and dislocate causing vertigo and balance problems ${ }^{3}$. The correct formation and anchoring of otoconia are essential for optimal vestibular function, balance, and the detection of sound ${ }^{3,6,7}$.

Zebrafish (Danio rerio) have become an increasingly valuable vertebrate model system in toxicology ${ }^{14,15}$ due to their high fecundity, external and transparent development, rapid generational times, reliance on evolutionarily conserved developmental regulatory gene networks, and sequenced genome, which facilitates mechanism-based toxicity studies. Like all vertebrate organisms, zebrafish have structural and sensory apparatus to perceive gravitational and positional cues for spatial awareness and posture control ${ }^{16}$, and perceive gravity and linear acceleration using mineralized biocrystals coupled to hair cells within the inner ear in a manner similar to other vertebrates, including humans. ${ }^{16}$ In zebrafish, these biocrystals are consolidated 
into three distinct and large structures termed otoliths that each directly interact with an entire patch of sensory epithelium located in the saccule, utricle, and a non-mammalian specific otolithic region known as the lagena ${ }^{6}$. The formation of otoliths in zebrafish has not yet been completely characterized but studies in trout and other species have shown that daily variations in the composition of vestibular endolymph are required ${ }^{8,9}$. This diurnal variation in the composition of the endolymph consists of increasing the amount of protein and decreasing the concentration of calcium and bicarbonate ions in the endolymph during the day and reversing this process at night. This variation allows the otoliths to grow as the fish increases in size, which is a unique characteristic of fish not thought to occur in other vertebrates. However, many of the major proteins responsible for otoconial development in mammals are required for otolith formation in zebrafish, including the regulatory protein Otop1, which is expressed in hair cells during the seeding and growth of otoconia and otoliths and is responsible for regulating protein secretion and movement of calcium to and from the endolymph ${ }^{3,18}$. This anatomical and molecular conservation together with their transparency and experimental tractability make zebrafish a robust tool to identify mechanisms underlying environmentally induced ototoxicity.

Cadmium (Cd), is a non-essential transition metal widely used in the production of batteries, solar panels, pigments, plastic stabilizers, and the production of other metals ${ }^{10}$. Oxidized cadmium $\left(\mathrm{Cd}^{2+}\right)$ typically enters the body through contaminated food and water as well as through inhalation of polluted air and cigarette smoke $e^{10,11}$. Exposure to $\mathrm{Cd}^{2+}$ has been shown to cause nephrotoxic, neurotoxic, osteotoxic, and carcinogenic effects $^{10,11}$, as well as ototoxicity (Agirdir et al. 2002; Kim et al., 2008, 
2009; Ozcaglar et al., 2001; Liu et al. 2014). Epidemiological studies have linked prenatal $\mathrm{Cd}^{2+}$ exposure to hyperactivity and balance disorders ${ }^{10}$ (Min et al., 2012), whereas in vivo animal studies have shown that $\mathrm{Cd}^{2+}$ accumulates within the vestibular system and leads to defective hearing (Ozcaglar et al, 2001). Although the molecular mechanisms underpinning the vestibular defects observed in both epidemiological and animal studies remain unknown, one study demonstrated damage to hair cells resulting from exposure to $\mathrm{Cd}^{2+}$ (Liu et al., 2014). We hypothesized that exposure to $\mathrm{Cd}^{2+}$ alters the composition of the endolymph leading to the production of abnormal otoconia/otoliths, which results in disruption of the otoconia/otolith-hair cell interaction, thus causing disorders characteristic of vestibular defects. In this study we show that zebrafish exposed developmentally to $\mathrm{Cd}^{2+}$ exhibit hyperactivity, circling behavior, altered otolith development and hair cell interactions, and have disrupted otop1 gene expression. Furthermore, supplementation with calcium rescues otolith formation and diminishes associated circling behavior but has no effect on the observed hyperactivity. These findings suggest that behavioral abnormalities resulting from $\mathrm{Cd}$ toxicity can be subdivided into disorders rooted in ototoxicity and disorders rooted in neurological impairment, each having a distinct mechanistic explanation. 


\section{Materials and Methods}

\section{Animal Husbandry}

Wildtype (AB strain) zebrafish were maintained at the NC State University

84

\section{7}

\section{Chemicals}

Stock solutions of cadmium chloride $\left(\mathrm{Cd}^{2+}\right.$, Cat. \# Sigma-Aldrich, $\left.\mathrm{MO}\right), \mathrm{N}-\mathrm{Acetyl}$ Cysteine (NAC, Cat. \# Sigma-Aldrich, MO), and calcium chloride (Cat. \# Sigma-Aldrich, $\mathrm{MO})$ were made in reagent-grade (Picopure ${ }^{\circledR}$ ) water at 10 parts-per-thousand $\left(\mathrm{CdCl}_{2}\right)$, $25 \mathrm{mg} / \mathrm{mL}(\mathrm{NAC})$ and $0.5 \mathrm{M}\left(\mathrm{CaCl}_{2}\right)$, and stored at room temperature in $1.5 \mathrm{~mL}$ polypropylene tubes. 10X embryo media (E2), consisting of $150 \mu \mathrm{M} \mathrm{NaCl}, 5 \mu \mathrm{M} \mathrm{KCl}, 10$ $\mu \mathrm{MgSO}_{4}, 1.5 \mu \mathrm{M} \mathrm{KH} \mathrm{PO}_{4}, 0.5 \mu \mathrm{M} \mathrm{Na}_{2} \mathrm{HPO}_{4}, 10 \mu \mathrm{M} \mathrm{CaCl}$, and $7 \mu \mathrm{M} \mathrm{NaHCO}$ was prepared in reagent-grade water and diluted to $0.5 \mathrm{X}$ for subsequent exposure studies.

\section{Exposures}

Zebrafish embryos were collected immediately after spawning and exposed to 30 - 60 parts-per-billion (ppb) $\mathrm{Cd}^{2+}$ in $0.5 \mathrm{X}$ E2 media from four hours post-fertilization (hpf) through seven days post-fertilization (dpf) at a density of 10 embryos $/ \mathrm{mL}$. This concentration range was selected as it represents the upper range of exposure observed in human populations exposed to cadmium-polluted environments ${ }^{2,11,21,22}$. The media was replaced daily and feeding began at five dpf. Cadmium has been shown to 
104 induce oxidative stress and interfere with ion channels, in particular zinc and calcium 105 channels ${ }^{24,25}$. To evaluate whether these effects were the primary cause of the 106 observed Cd-induced otolith and/or behavioral phenotypes, embryos were co-exposed 107 from four hpf to $7 \mathrm{dpf}$ with daily media changes to $\mathrm{Cd}^{2+}(60 \mathrm{ppb})$ and either $1 \mathrm{mg} / \mathrm{L} \mathrm{N}$ acetyl-L-cysteine (NAC) or $2.5 \mathrm{mM}$ Calcium $\left(\mathrm{CaCl}_{2}\right)$.

\section{Otolith Imaging and Measurements}

112 microscope. To assess otolith size, seven dpf zebrafish larvae $(n=5)$ were anesthetized 113 by the addition of phosphate-buffered Tricaine (final concentration: $0.4 \mathrm{mg} / \mathrm{L}, \mathrm{pH}$ 7, 114 Western Chemical Inc.) to 0.5X E2 media until no movement was observed. Once 115 anesthetized, images were taken using the Leica Application Suite (software version 4.8.0). The length of the otoliths, which resemble prolate spheroids, was determined by

117 measuring across the longest axis using the GNU Image Manipulation Programs 118 protractor tool (version 2.8.18) ${ }^{23}$.

\section{Scanning Electron Microscopy}

122 triplicate ( $\mathrm{n}=10$ per replicate) and placed on ice for 10-15 minutes to euthanize them.

123 Larvae were dehydrated using successive 15-minute incubations in a graded ethanol

124 series (33\%, 66\%, and 100\%), and otoliths were dissected using fine-tipped forceps

125 under a Leica dissecting microscope and stored in $100 \%$ ethanol at $4^{\circ} \mathrm{C}$ for a maximum

126 of seven days. Otoliths were fixed in $3 \%$ glutaraldehyde in $0.1 \mathrm{M} \mathrm{NaPO}$ buffer

127 (phosphate buffer), $\mathrm{pH} 7.4$ at $4^{\circ} \mathrm{C}$ and afterwards transferred to microporous specimen 
128 capsules (Structure Probe Inc., West Chester PA) for processing. Samples were 129 washed in three changes of phosphate buffer and dehydrated in a graded ethanol 130 series to $100 \%$ before critical point drying in liquid $\mathrm{CO}_{2}$ (Tousimis Research 131 Corporation, Rockville MD). Otoliths were placed on carbon tabs (Ladd Research 132 Industries, Williston VT) and sputter coated with Au/Pd using a Hummer 6.2 sputter 133 system (Anatech USA, Union City CA). Samples were viewed on a JEOL JSM-5900LV 134 at $15 \mathrm{kV}$ (JEOL USA, Peabody MA).

\section{Behavioral Assays} (Noldus; Leesburg, VA). Behavior analyses for all exposures were conducted at five dpf. Zebrafish larvae were arrayed in a six-well plate at a density of eight larvae per well.

140 Plates were placed in the DanioVision ${ }^{\mathrm{TM}}$ box and larvae were allowed to acclimate in 141 the dark for at least 30 minutes. Following acclimation, larval response to three 10-

142 minute dark-light cycles was measured. Measured end points included movement, 143 acceleration, velocity, and rotation, including binning into clockwise or counterclockwise 144 rotation. As zebrafish larvae are more active in the dark, cumulative distance moved in 145 the dark was used to compare treated vs. controls.

\section{Quantitative PCR}

Larvae ( $\mathrm{n}=10$ per replicate) were pooled in triplicate at $6,12,18,24,36,48$, and

$149120 \mathrm{hpf}$ into $1.5 \mathrm{~mL}$ polypropylene tubes and euthanized by placement on ice for 10 150 minutes. All liquid was removed and $800 \mu \mathrm{L}$ of TriReagent (Sigma-Aldrich, MO) was 
151 added to each tube. Larvae were homogenized using RNase-free disposable

152 micropestles. Total RNA was extracted according to the manufacturer's protocol and the

153 integrity and concentration were assessed on an Agilent Bioanalyzer using the Agilent

154 RNA 6000 Nano Kit (Agilent Technologies, CA). Equal amounts of total RNA from each

155 sample were used to make cDNA by reverse transcription PCR using a poly-dT primer.

156 Quantitative PCR was performed using SYBR Green and ROX reference dye (Agilent

157 Technologies, CA). Primer sequences for star marker (stm), secreted protein, acidic,

158 cysteine-rich (sparc), otopetrin 1 (otop1), and eukaryotic translation elongation factor 1

159 alpha 1, like 1 (eef1a1/1) are provide in Table 1. All qPCR assays were normalized to

160 eef1a1/1, which did not vary across treatment groups. Primer pairs were designed to

161 exons separated by at least one intron to avoid amplifying genomic DNA. The $2^{\wedge}(-$

$162 \Delta \triangle \mathrm{CT}$ ) method was used to approximate relative transcript fold change between

163 treatment groups ${ }^{24}$. 


\section{Results}

\section{Cadmium Induces Changes in Otolith Diameter}

Developmental cadmium exposure resulted in a decrease in the diameter of the saccule otolith at $7 \mathrm{dpf}$ relative to untreated controls (Figure 1); however, the utricle otolith diameter was not significantly different between controls and Cd-exposed larvae. The decrease in saccule otolith also exhibited a dose-dependent decrease beginning at

$17130 \mathrm{ppb}(p<0.01)$ and leveling off at $40 \mathrm{ppb}$ cadmium $(p<0.001)$ (Fig. 1d). These

172 results demonstrate that developmental $\mathrm{Cd}^{2+}$ exposure reduces the size of the saccule 173 otolith in larval zebrafish without affecting the utricle otolith.

\section{Cadmium Alters Otolith Ultrastructure}

To assess the ultrastructure of the saccule otoliths, we used scanning EM (SEM)

177 to visualize control and Cd-exposed saccule otoliths. Our initial analysis revealed 178 pronounced structural differences in the Cd-exposed otoliths compared to control 179 otoliths. Whereas, control otoliths exhibited a smooth surface with evidence of otolith 180 attachment to hair cell stereocilia, Cd-exposed otoliths exhibited a course knobbled 181 surface with numerous fiber or cable-like extensions between knobs and an absence of 182 evidence of attachment to hair cells (Fig. 2). The presence of numerous cable-like extensions and the pronounced increase in surface area due to surface knobbling in the 184 Cd-exposed otoliths suggests a defect in the deposition of inorganic minerals or specific 185 proteinaceous components required for normal otolith formation. 
To determine if the otolith phenotype observed in Cd-exposed zebrafish correlated with changes in behavior, we assessed several behavioral endpoints,

196 increased the number of rotations in both a clockwise (CW) and counter clockwise

197 (CCW) direction but with a clear preference for CCW movement $(p<0.0001$; See 198 Supplemental Video). This circling behavior is characterized by a rapid twirling or spinning behavior in response to both mechanical and light stimuli and is often observed in other model organisms harboring mutations that affect the vestibular system ${ }^{27,28}$.

201 These data demonstrate that $\mathrm{Cd}^{2+}$ induces a dose-dependent increase in activity and 202 number of rotations in 5 dpf larval zebrafish.

\section{Calcium Rescues Otolith Formation and Diminishes Vestibular-Related Rotational}

\section{Behavior but not Hyperactivity}

Cadmium has been shown to interfere with divalent ion channel function, in

207 particular zinc channels and both voltage and non-voltage gated calcium channels, and

208 to induce oxidative stress ${ }^{29,30}$. To test the hypothesis that $\mathrm{Cd}^{2+}$ interferes with calcium

209 homeostasis in the vestibular system, zebrafish embryos were co-exposed to $\mathrm{Cd}^{2+}$ and 210 the effect of exogenous calcium on otolith formation was assessed. As seen in Figure 4, 
211 calcium prevented Cd-mediated decrease in saccule otolith size. In addition, the

212 inclusion of calcium in the media was associated with a decrease in the rotational

213 behavior observed in presence of $\mathrm{Cd}^{2+}$ only, indicating that the defects in otolith

214 formation were inducing behavioral effects commonly observed in other model

215 organisms with vestibular defects. However, the addition of calcium was not effective at

216 reducing hyperactivity (Fig 4). In fact, addition of calcium alone induced a hyperactive

217 phenotype independent of $\mathrm{Cd}^{2+}$ exposure. To test the hypothesis that changes in otolith

218 size and hyperactivity were due to $\mathrm{Cd}^{2+}$-dependent increases in reactive oxygen

219 species (ROS), a phenomenon reported in numerous studies ${ }^{13}$, we exposed larvae to

$220 \mathrm{Cd}^{2+}$ alone or in combination with NAC, a ROS scavenger, Whereas NAC was able to

221 rescue hyperactivity, it did not prevent the decrease in saccule otolith size nor the

222 increased circling behavior induced by $\mathrm{Cd}$ exposure (Fig 4). These results provide

223 evidence that Cd-induced hyperactivity and circling behaviors are independent events

224 likely due to the disruption of different molecular pathways, including vestibular (circling)

225 and neurological (hyperactivity) pathways, and that hyperactivity is not dependent on

226 otolith malformations and associated vestibular defects.

228 Cadmium Induces Changes in mRNA expression of the Otolith Protein, otop1

230 the calcium regulatory gene otop 1 is significantly affected by exposure to $\mathrm{Cd}^{2+}$ during

231 early development. At 6 and 24 hpf, otop1 gene expression was significantly $(p<0.05)$

232 reduced in Cd-exposed versus unexposed embryos (Fig 5). This trend reversed at 36

233 hpf at which point it was significantly $(p<0.05)$ elevated in Cd-exposed embryos. Otolith 
234 genes that encode for the structural proteins Sparc, and Stm were not significantly

235 altered at any time points tested. These data demonstrate that zebrafish

236 developmentally exposed to $\mathrm{Cd}^{2+}$ exhibit mRNA expression changes in otop1, a gene

237 important for vestibular calcium sensing and signaling. 


\section{Discussion}

Developmental exposure to $\mathrm{Cd}^{2+}$ reduces the size of the saccule otolith in a

241 dose-dependent manner. This decrease was characterized by a $21 \%$ reduction in the

242 size of the saccule otolith (Fig 1) at 40, 50, and $60 \mathrm{ppb} \mathrm{Cd}^{2+}$. SEM revealed that not only

243 was the saccule otolith reduced in size but that there are significant changes in the

244 ultrastructure. Otoliths exposed to $\mathrm{Cd}^{2+}$ have a rough cauliflower surface texture

245 whereas the control otoliths are relatively smooth (Fig 2). We observe that $\mathrm{Cd}^{2+}$ induced

246 hyperactivity in a dose-dependent manner by assessing average total distance moved

247 in the dark (Fig 3) and that calcium is capable of rescuing otolith formation but not

248 hyperactivity (Fig 3, Fig 4). Calcium alone is capable of inducing hyperactivity which

249 might account for why it only rescues otolith formation. NAC was able to reduce

250 cadmium-induced hyperactivity but could not rescue otolith formation (Fig 3, Fig 4)

251 Semi-quantitative qPCR analysis revealed that $\mathrm{Cd}^{2+}$ altered the expression level

252 of otop1, a gene important for vestibular calcium sensing and transport. More work is

253 needed to determine whether or not the $\mathrm{Cd}^{2+}$-induced reduction in saccule otolith size is

254 responsible for the observed hyperactivity and/or rotation phenotype. The ultrastructure

255 showed that the $\mathrm{Cd}^{2+}$-exposed saccule otoliths were missing structural elements and

256 when combined the observations of changes in otop1 gene expression hints at a

257 possible loss of calcium deposits. Further the cable-like structure seen in the SEM

258 images may be a type of structural or scaffolding element required during the

259 biosynthesis of the otoliths. To help answer these questions we intend to test additional

260 ROS scavengers ${ }^{31}$, evaluate the uptake of $\mathrm{Cd}^{2+}$ using a $\mathrm{Cd}-109$ radiotracer in the

261 presence and absence of potentially protective compounds such as ebselen, calcium, 
262 and ascorbic acid, determine the protein and calcium composition of the saccule otoliths

263 using Inductively coupled plasma mass spectrometry (ICP-MS), and determine the

264 mechanism by which $\mathrm{Cd}^{2+}$ disruptions otop1 and its effects on otolith development.

265 Hyperactive behavioral disorders were estimated to affect between 5-11\% for

266 children between 4-17 years of age in 2011 (6.4 million $)^{32}$. The data we have presented

267 provides a plausible mechanistic link between an inner ear defect and hyperactivity

268 because of $\mathrm{Cd}^{2+}$-exposure. By using common fairly innocuous compounds, as

269 demonstrated here, it may be possible to reduce the number of children born with

270 ADHD in the future. 


\section{References}

1. Sahmoun, A. E., Case, L. D., Jackson, S. A. \& Schwartz, G. G. Cadmium and Prostate Cancer: A Critical Epidemiologic Analysis. Cancer Invest. 23, 256-263 (2005).

2. ATSDR. TOXICOLOGICAL PROFILE FOR CADMIUM. (2012).

3. Lundberg, Y. W., Xu, Y., Thiessen, K. D. \& Kramer, K. L. Mechanisms of otoconia and otolith development. Dev. Dyn. 244, 239-253 (2015).

4. Abbas, L. \& Whitfield, T. T. in Fish Physiology 29, 123-171 (Elsevier, 2010).

5. Yoon, T. H., Paparella, M. M. \& Schachern, P. A. Otosclerosis involving the vestibular aqueduct and Meniere's disease. Otolaryngol. Neck Surg. 103, 107-112 (1990).

6. Inoue, M., Tanimoto, M. \& Oda, Y. The role of ear stone size in hair cell acoustic sensory transduction. Sci. Rep. 3, (2013).

7. Cruz, S., Shiao, J.-C., Liao, B.-K., Huang, C.-J. \& Hwang, P.-P. Plasma membrane calcium ATPase required for semicircular canal formation and otolith growth in the zebrafish inner ear. J. Exp. Biol. 212, 639-647 (2009).

8. Borelli, G. Daily variations of endolymph composition: relationship with the otolith calcification process in trout. J. Exp. Biol. 206, 2685-2692 (2003).

9. Campana, S. E. Chemistry and composition of fish otoliths: pathways, mechanisms and applications. Mar. Ecol. Prog. Ser. 188, 263-297 (1999).

10. Roth, J. A. \& Salvi, R. Ototoxicity of Divalent Metals. Neurotox. Res. (2016). doi:10.1007/s12640-016-9627-3

12. Antoine, M. W., Hubner, C. A., Arezzo, J. C. \& Hebert, J. M. A Causative Link Between Inner Ear Defects and Long-Term Striatal Dysfunction. Science 341, 11201123 (2013). 
13. Kim, S.-J. et al. The Protective Mechanism of Antioxidants in Cadmium-Induced Ototoxicity in Vitro and in Vivo. Environ. Health Perspect. 116, 854-862 (2008).

14. Planchart, A. et al. Advancing toxicology research using in vivo high throughput toxicology with small fish models. ALTEX 33, 435-452 (2016).

15. Bugel, S. M., Tanguay, R. L. \& Planchart, A. Zebrafish: A marvel of highthroughput biology for 21(st) century toxicology. Curr. Environ. Health Rep. 1, 341352 (2014).

16. Söllner, C. \& Nicolson, T. in Biomineralization (ed. Bäuerlein, E.) 229-242 (Wiley-VCH Verlag GmbH \& Co. KGaA, 2005).

17. Xu, Y., Zhang, Y. \& Lundberg, Y. W. Spatiotemporal differences in otoconial gene expression. genesis (2016). doi:10.1002/dvg.22990

18. Kim, E. et al. Regulation of Cellular Calcium in Vestibular Supporting Cells by

Otopetrin 1. J. Neurophysiol. 104, 3439-3450 (2010).

19. Go, W., Bessarab, D. \& Korzh, V. atp2b1a regulates Ca2+ export during Calcium 48, 302-313 (2010).

20. Westerfield, M. The zebrafish book. A guide for the laboratory use of zebrafish

314 21. Ibrahim, K. S., Beshir, S., Shahy, E. M. \& Shaheen, W. Effect of Occupational 315 Cadmium Exposure on Parathyroid Gland. Open Access Maced. J. Med. Sci. 4, 302 316 (2016). removal from aqueous solutions. Int. J. Eng. Sci. Technol. 2, (2010). 
23. GIMP. GIMP Available at: https://www.gimp.org/. (Accessed: 27th February 2017)

24. Livak, K. J. \& Schmittgen, T. D. Analysis of relative gene expression data using real-time quantitative PCR and the 2(-Delta Delta C(T)) Method. Methods San Diego Calif 25, 402-408 (2001).

25. Burgess, H. A. \& Granato, M. Modulation of locomotor activity in larval zebrafish during light adaptation. J. Exp. Biol. 210, 2526-2539 (2007).

26. MacPhail, R. C. et al. Locomotion in larval zebrafish: Influence of time of day, lighting and ethanol. NeuroToxicology 30, 52-58 (2009).

27. Lv, K. et al. Circling behavior developed in Dmp1 null mice is due to bone defects in the vestibular apparatus. Int J Biol Sci 6, 537-545 (2010).

28. Hurle, B. Non-syndromic vestibular disorder with otoconial agenesis in tilted/mergulhador mice caused by mutations in otopetrin 1. Hum. Mol. Genet. 12, 777-789 (2003).

29. Hinkle, P. M., Kinsella, P. A. \& Osterhoudt, K. C. Cadmium uptake and toxicity via voltage-sensitive calcium channels. J. Biol. Chem. 262, 16333-16337 (1987).

30. Komjarova, I. \& Bury, N. R. Evidence of Common Cadmium and Copper Uptake Routes in Zebrafish Danio rerio. Environ. Sci. Technol. 48, 12946-12951 (2014).

31. Rousset, F., Carnesecchi, S., Senn, P. \& Krause, K.-H. NOX3-TARGETED THERAPIES FOR INNER EAR PATHOLOGIES. Curr. Pharm. Des. 21, 5977-5987 (2015). 
340 32. Attention-Deficit / Hyperactivity Disorder (ADHD) || CDC. Attention-Deficit /

341 Hyperactivity Disorder (ADHD) / NCBDDD / CDC Available at:

342 https://www.cdc.gov/ncbddd/adhd/index.html. (Accessed: 31st March 2017)

343

344 
345 Table 1. PCR Primer Sequences Used for qPCR

\begin{tabular}{|l|l|l|}
\hline Gene & Forward primer & Reverse primer \\
\hline stm & AAGCCATTGAGGTCGTGGAG & GCAGGGAGCGATGTATTTGC \\
\hline sparc & AAGCCATTGAGGTCGTGGAG & GCAGGGAGCGATGTATTTGC \\
\hline otop1 & CATCGGGTATTTCGTGGGCT & CATCTCGATCAGCCGTCTCC \\
\hline eef1a1/1 & TACAAATGCGGTGGAATCGAC & GTCAGCCTGAGAAGTACCAGT \\
\hline
\end{tabular}

346 
1 Figure 1: Otolith Size Decreases in Response to $\mathbf{C d}^{2+}$ Exposure. (A) Zebrafish dose

2 response to an exposure of $30-60$ parts per billion (ppb) Cadmium $\left(\mathrm{Cd}^{2+}\right) .(\mathrm{B})$

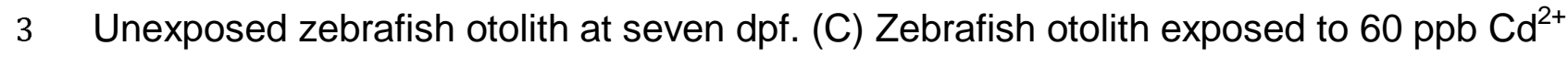

4 at seven dpf. (D) Diameter of zebrafish saccule otolith at seven dpf. saccule otolith (S),

5 utricle otolith $(U) ;{ }^{* *} p<0.01,{ }^{* * *} p<0.001$.

6 Figure 2: Saccule Otolith SEM Images at Seven dpf. (A) Unexposed zebrafish otolith.

(B) Zebrafish otolith exposed to $60 \mathrm{ppb} \mathrm{Cd} 2+$. (C) High magnification image of

8 unexposed otolith. (D) High magnification image of otolith exposed to $60 \mathrm{ppb} \mathrm{Cd} 2+$. (E)

9 Very high magnification image of unexposed otolith. (F) High magnification image of

10 otolith exposed to $60 \mathrm{ppb} \mathrm{Cd} 2+$. Study design for the collection for zebrafish saccule

11 otolith to assess $\mathrm{Cd} 2+$ induced changes in ultrastructure using scanning electron

12 microscopy (SEM).

13 Figure 3: $\mathbf{C d}^{2+}$ Induced Hyperactivity and Rotations. (A) Zebrafish distance moved

14 in response to light and dark cycling. (B) Number of clockwise and counter clockwise

15 rotations in the dark in response to $\mathrm{Cd}$. (C) Average total distance moved in the dark in

16 response to $\mathrm{Cd}$. (D) Average total distance moved in the dark in response to rescue

17 treatments. (A-D) Behavioral assessments made using Noldus Ethovision software; * $p$

$18<0.05,{ }^{* * * *} \mathrm{p}<0.0001$.

19 Figure 4: Otolith Recovers when Treated with Ca. (A) Otolith rescue experiments

20 using $1 \mathrm{mg} / \mathrm{L} \mathrm{N}$-Acetyl Cysteine (NAC) or $2.5 \mathrm{mM}$ Calcium. (B) Changes in saccule

21 otolith diameter relative to water controls with rescue treatments; ${ }^{* *} p<0.01$. 
22 Figure 5: Quantitative PCR of Otolith Genes from 4 - 120 Hours Post-Fertilization.

23 (A) Otopetrin 1 (otop1) gene expression. (B) Starmaker (stm) gene expression. (C)

24 Secreted protein, acidic, cysteine-rich (sparc) gene expression; * $p<0.05$. 


\section{$25 \quad$ Figure 1}
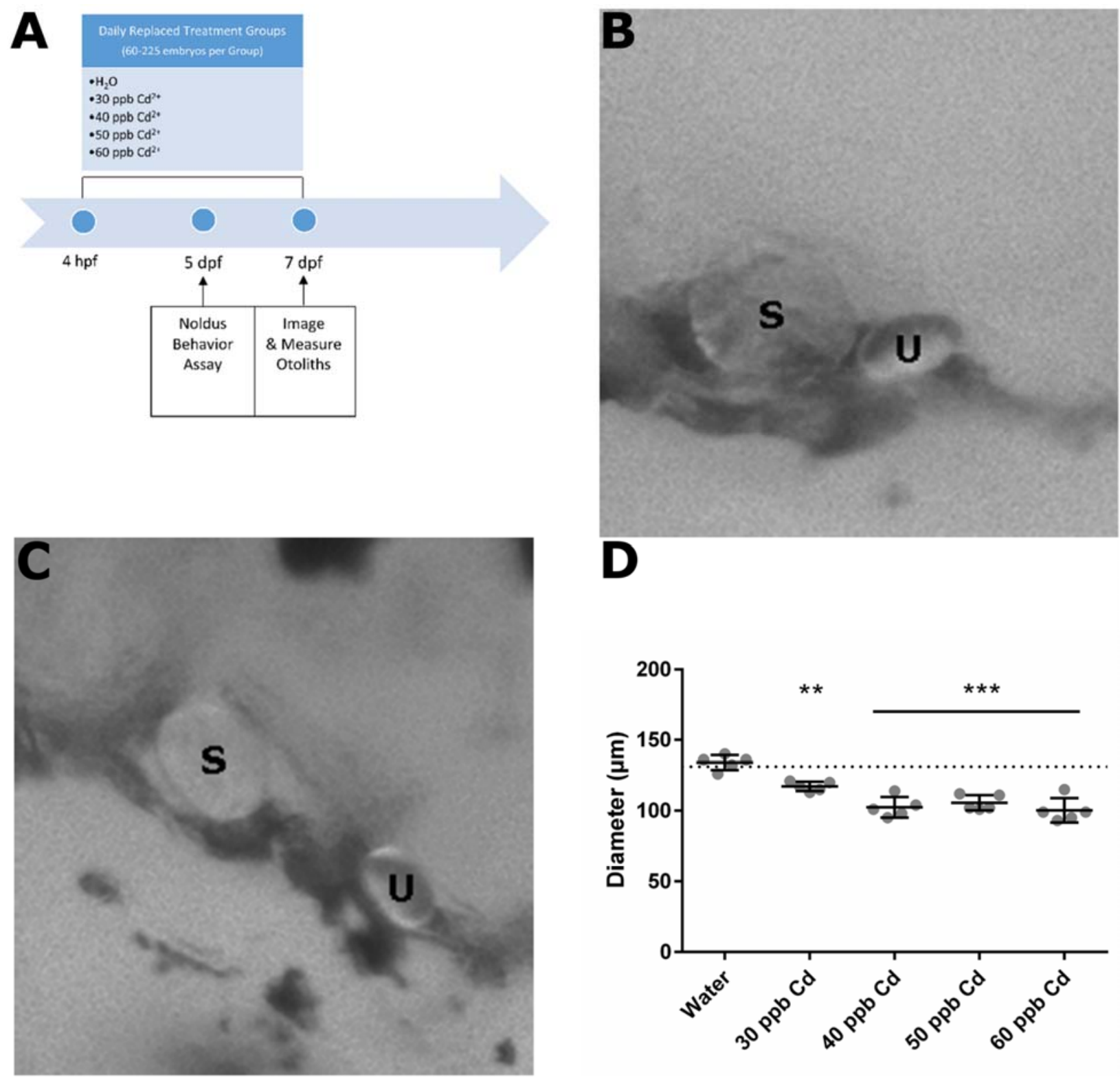

D

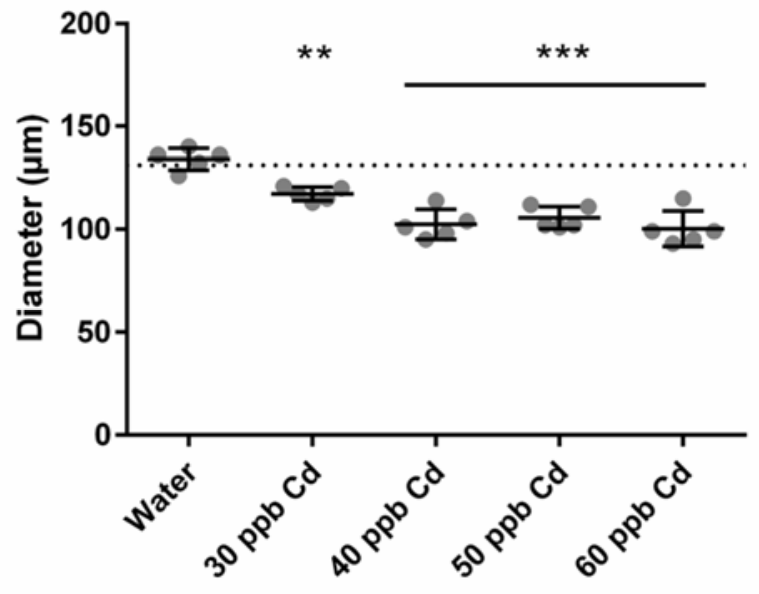




\section{Figure 2}
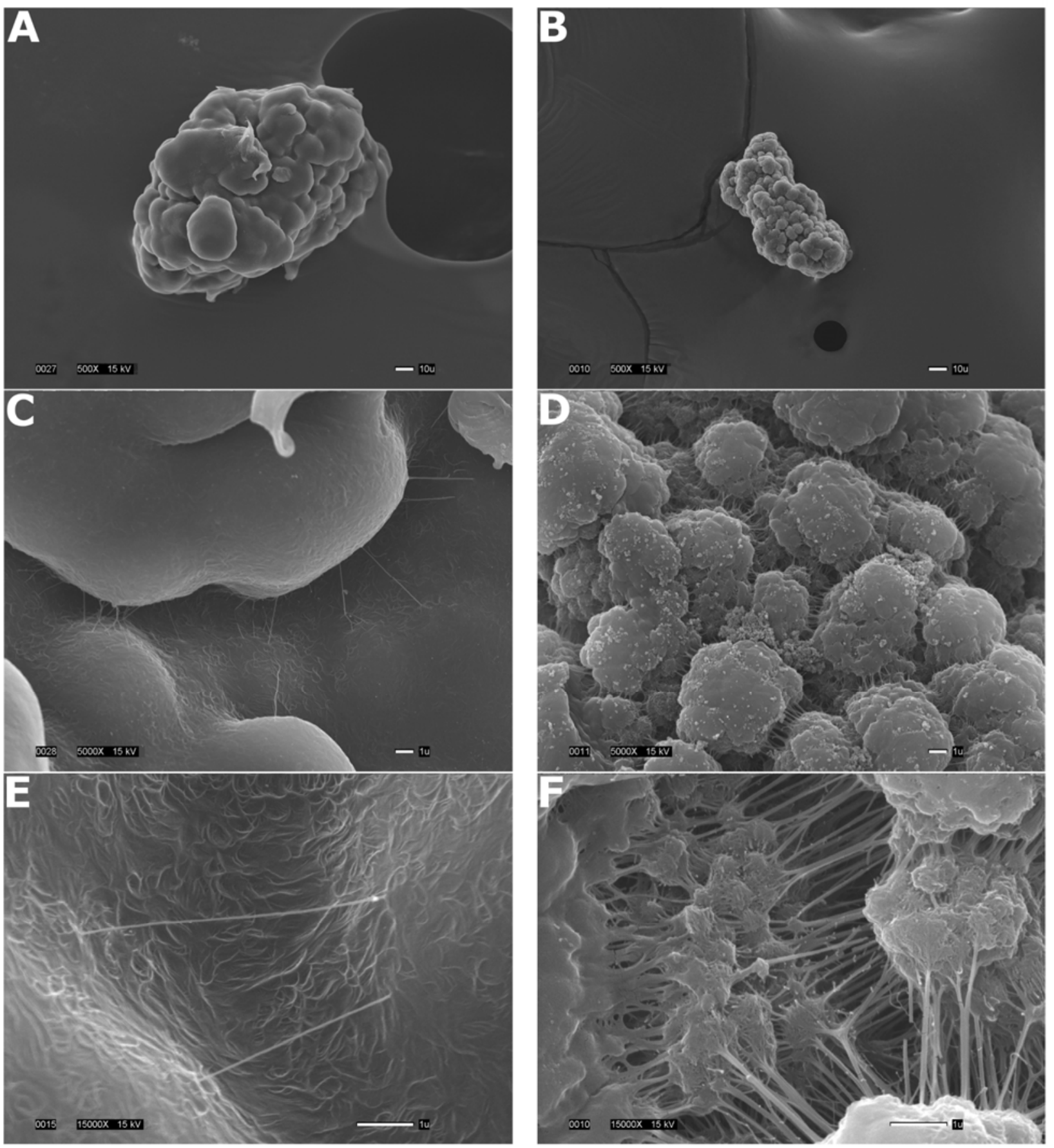
A
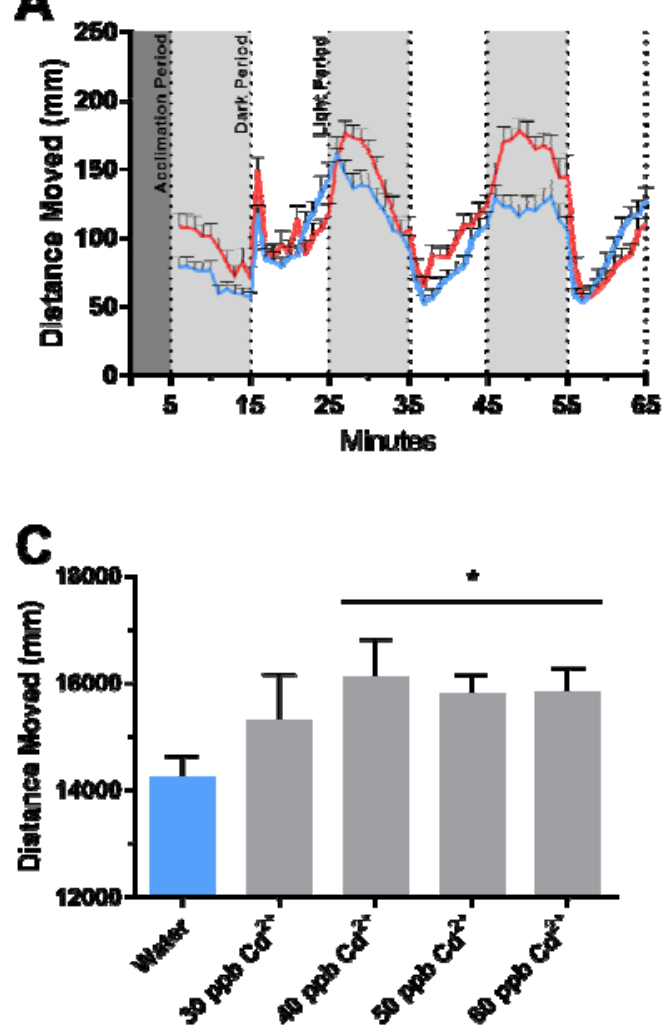

B
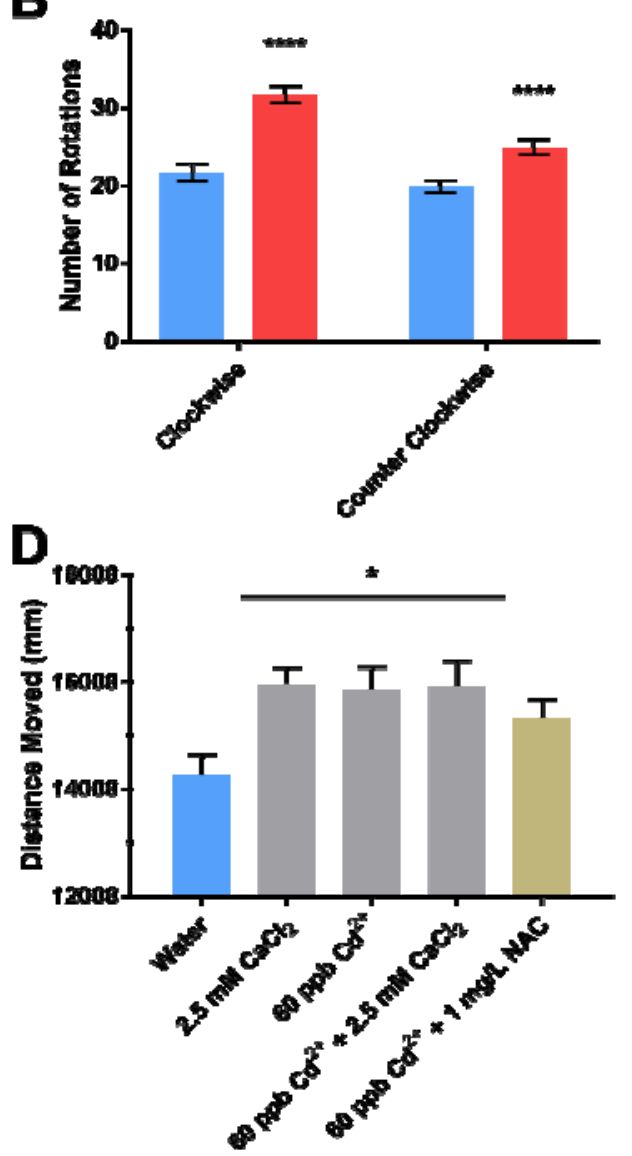
Figure 4

A

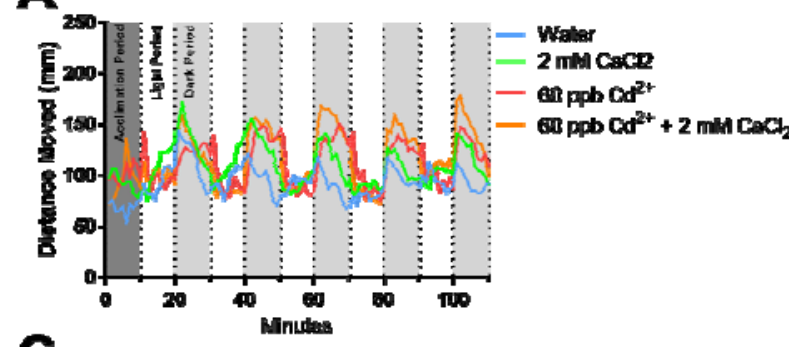

C

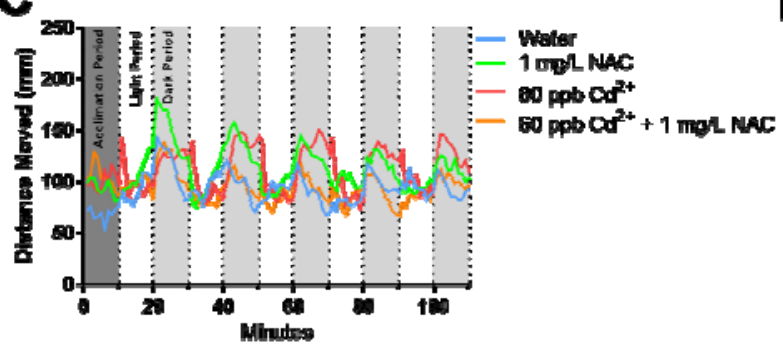

B
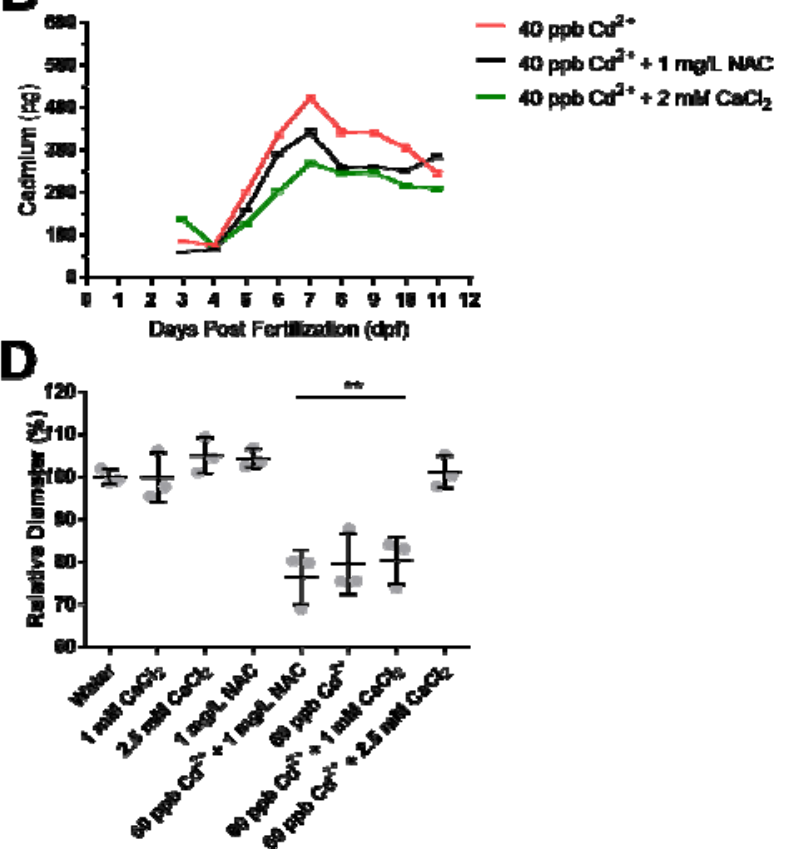


\section{$33 \quad$ Figure 5}
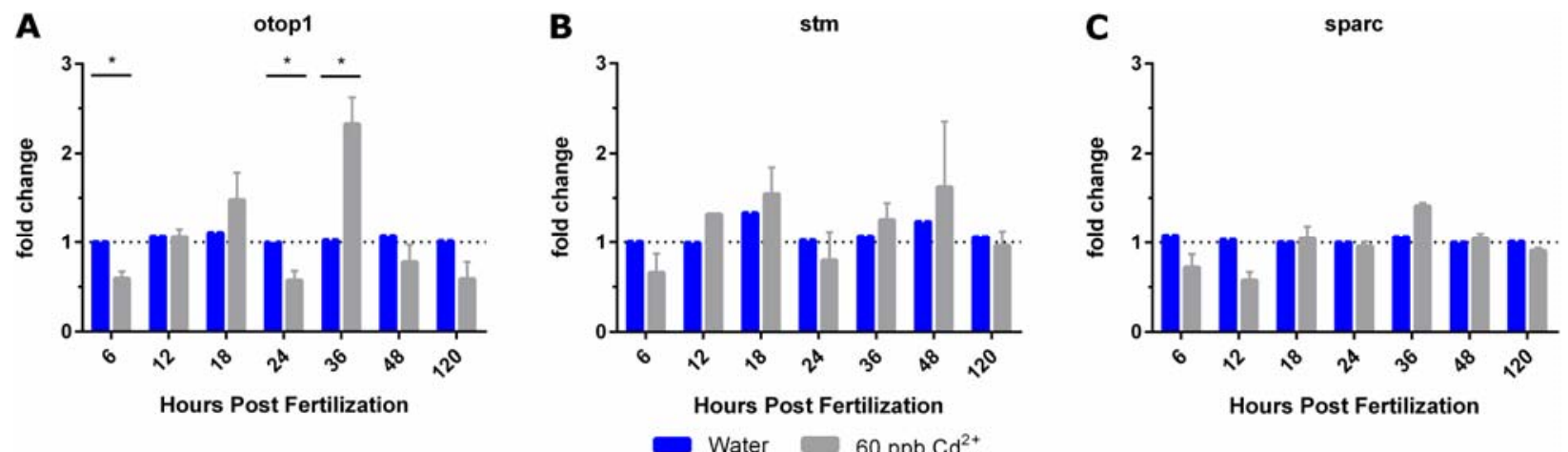